https://doi.org/10.18778/2196-8403.2021.03

\title{
Filmallegorien der Nationen. Über die Position der Zuschauenden
}

Das Kino hat neue Formen der Kollektivität geschaffen, nämlich einen identitätspolitischen und affektiven Anschluss der Zuschauenden an einen Staat, eine Nation und die Macht der Geschichte. Dieser Anschluss wird zunächst mit dem Neologismus Staatsgenre diskutiert, um die sozialpolitische Hierarchisierung der Genres und deren Produktionsund Rezeptionsbedingungen zu konzeptualisieren. In Anlehnung an feministische Theorien der Zuschauenden wird daraufhin versucht, eine Sehperspektive zu erfassen, die an der Gemeinschaftsbildung beteiligt wird. In diesem Zusammenhang wird zum einen der narratologische Ansatz von Teresa de Lauretis interessant, mit dem Identifikationsprozesse mit dem Bild beschrieben werden. Zum anderen wird die Allegorie als eine affektive Figur gedacht, mit der die Zuschauenden performativ eine exzentrische Position erlangen bzw. mit der Abstrahierung des Bildes zur Allegorie die Umwandlung mitvollziehen. Diesen Prozessen können sich die Zuschauenden nicht entziehen, weil es um deren affektive Überwältigung durch die Bilder geht, die u.a. durch die Auflösung der Gendersemantiken bedingt wird. Als Beispiel fungiert der oscarprämierte Blockbuster Saving Private Ryan (USA 1998).

Schlüsselwörter: Staatsgenre, nationale Allegorien, Affektbild, Erinnerungskulturen, feministische Blicktheorien

\section{National allegories in film. The viewers' perspective}

Cinema has created new forms of collectivity, namely identity-political and affective connections between the viewers and the state, nation, and the power of history. The article discusses this connection using the "state genre" neologism. This enables the Author to place the film genre within the socio-political hierarchy and in the context of film production and reception. Based on feminist theories of spectatorship, an attempt is then made to capture a viewer's perspective as part of community formation. In this context, Teresa de Laurentis' narratological approach is of interest, as it describes the process of building identity through the image. As an affective figure, the allegory also seems relevant, as it enables the viewers to attain some perspective on the projected images 
- or to modify their opinions, as they perceive the images to form an allegory. The viewers allow for this process to happen, and to be overpowered by the images (this is conditioned, among others, by the dissolution of gender semantics). The Oscar-winning blockbuster Saving Private Ryan (USA 1998) is presented as an example.

Keywords: State genre, national allegories, affect image, memory cultures, feminist theories of the gaze

\section{Narodowe alegorie filmowe. $O$ perspektywie widza}

Kino stworzyło nowe formy wspólnotowe: związek z państwem, narodem i historią opierający się na politycznej i emocjonalnej tożsamości widzów. Związek ten rozpatrywany jest w artykule za pomocą neologizmu ,gatunek narodowy“. Umożliwia to usytuowanie gatunku filmowego w hierarchii społeczno-politycznej oraz w kontekście produkcji i recepcji dzieła filmowego. W oparciu o teorie feministyczne podjęta zostaje próba uchwycenia perspektywy widza w procesie tworzenia wspólnoty. Interesująca w tym kontekście okazała się propozycja interpretacji narratologicznej Teresy de Lauretis, opisującej proces budowania tożsamości pod wpływem mowy obrazów. Z drugiej strony jako figura afektywna rozumiana jest alegoria, dzięki której widzowie nabierają dystansu do oglądanej treści lub też modyfikują swą postawę, ponieważ traktują obraz jako alegorię. Widzowie ulegają temu procesowi, ponieważ chodzi w nim o afektywne poddanie się obrazom, co jest między innymi uwarunkowane rozpadem semantyki płci. Jako przykład służy nagrodzony Oskarem hit filmowy Szeregowiec Ryan (Saving Private Ryan, USA 1998).

Słowa klucze: gatunek narodowy, alegorie narodowe, obrazy afektywne, kultury pamięci, feministyczne teorie postrzegania

\section{Was haben feministische Blicktheorien vergessen?}

Feministische Debatten zur Position der Zuschauenden gehören eigentlich in die Wissenschaftsgeschichte, scheinen mir jedoch produktiv erweiterbar und aktualisierbar zu sein. Vor allem haben die feministischen Blicktheorien das zentrale strukturierende Moment des Hollywood-Cinema vergessen: seine Genrehaftigkeit. Diese wurde in der Regel in verschiedenen theoretischen Ansätzen en passant erwähnt, jedoch in Bezug auf entsprechende Blickstrukturen nicht differenziert (vgl. z. B. MULVEY 1994; DOANE 1985). Bevorzugt wurden einzelne Genres, die das Individuelle verhandeln. Als analytisches Instrumentarium diente dabei die Psychoanalyse, deren Perspektive auf (De-)Subjektivierungsprozessen liegt und die diesen Fokus teilweise vorbestimmt haben mag. Im Kontext der Filmwissenschaften schlug die Psychoanalyse sich in der Konzipierung individueller Rezeptionshaltungen nieder. Dadurch wurden jene Prozesse außer Acht gelassen, mit denen Individuen an gesellschaftliche Diskurse, zum Beispiel an die Geschichte des Staates oder an die Macht der Insti- 
tutionen, angeschlossen werden, die vor allem durch Massenmedien möglich werden.

BENEDICT ANDERSON (1996) zeigt in seiner berühmten Studie, dass die Nation u.a. dank der Zeitung als Einheit vorstellbar wurde. Die Zeitungen stellten einen gemeinsamen Wissenshorizont her, der sich auf den Staat und seine Bürger*innen bezog und diese so miteinander verband (vgl. ANDERSON 1996:68). ${ }^{1}$ Darüber hinaus wurden Zeitungen rituell, also immer zur gleichen Zeit, in der Regel abends und morgens gelesen, sodass die nationale Gemeinschaft als Gemeinschaft der Lesenden performativ und in der Zeit produziert wurde. Im Laufe des 20. Jahrhunderts werden diese Funktionen mehr und mehr durch den Film und das Fernsehen übernommen. Filme haben aufgrund ihrer spezifischen Medialität Erinnerungskulturen in der aktuellen Form hervorgebracht, die den Staat neu zu denken ermöglichen - in Form einer Art kompakten, affektiven, zu handhabenden Biografie, an die sich Individuen anschließen können (vgl. GRADINARI 2020). Mehrere Studien heben die Wirksamkeit des Films als Gedächtnismedium hervor (vgl. KAES 1987:208; ROSENSTONE 1993:200; ELSAESSER 2002:20; WelZER / MOLLER / TSCHUGGNALL 2002; WeLZER 2005), beschreiben jedoch nicht, wie es nun möglich ist, das Individuelle und das Kollektive zusammenzudenken: Wie also kann die kollektive Historie in das eigene Selbstbild integriert werden?

Dieser Frage möchte ich mich zunächst mit dem Begriff des Staatsgenres annähern, dem jene Filme mit großer politischer Wirkung und in der Regel auch mit einem großen Budget zugeordnet werden. Die zentrale Strategie dieser Filme ist dabei, Figuren zu allegorisieren, womit sie über ein individuelles Schicksal erhoben werden und so als Verkörperung der Nation fungieren. Durch Allegoriebilder lässt sich daher die Position der Zuschauenden neu denken, die in Anlehnung an TERESA DE LAURETIS (1990) und in Bezug auf Affekttheorien diskutiert wird. Allerdings geht es in diesem Beitrag nicht um eine empirische Rezeptionserfahrung, sondern - ganz im Sinne früherer feministischer Theoriebildung - um eine Beschreibung filmischer Strukturen, die eine solche Position der Zuschauenden medienästhetisch herausbilden. Als Beispiel fungiert der mehrfach ausgezeichnete, viel gepriesene und Oscar-gekrönte Kriegsfilm Saving Private Ryan [Der Soldat James Ryan] (USA 1998, R.: Steven Spielberg).

1 In diesem Beitrag verwende ich Nation und Staat synonym, da beide im Film oft zusammenfallen. Grundsätzlich bedarf es allerdings es einer genaueren und differenzierten Untersuchung, die hier vernachlässigt werden muss. 


\section{Staatsgenre und Staatsfilm}

Um das Verhältnis zwischen einem Staat und Genres zu beschreiben, möchte ich in Anlehnung an den barocken Staatsroman den Begriff des Staatsgenres vorschlagen. Mit diesem Begriff kann ebenso eine gesellschaftliche Priorisierung der Filme wie deren politisches Potenzial durch den Staat beschrieben werden, da er es ermöglicht, auf die kulturelle Relevanz der Genres aufmerksam zu machen. RAPHAËLLE MOINE (vgl. 2008:189) schreibt in diesem Kontext von Genre-Regimen. Sie organisieren Produktion und Rezeption von Genres hierarchisch. Einige politisch oder gesellschaftlich bevorzugte Genres werden entsprechend besser finanziert oder sogar direkt durch den Staat gefördert; diese beeinflussen aufgrund der angewachsenen Bedeutsamkeit alle weiteren Genres. Es geht also um die ideologische Wirksamkeit der Genres (vgl. GAMPER 2010:68), die jedoch nicht (allein) über die ,Botschaft' auszumachen ist - filmische Genres verdichten vorherrschende Ideologeme (vgl. JAMESON 1988) und transformieren diese in Affekte, die Subjektstrukturen prägen, zum Beispiel in Form des prothetischen Gedächtnisses (vgl. LANDSBERG 2016:20f).

Genreregime totalitärer Staaten unterscheiden sich dabei grundsätzlich von demokratisch-kapitalistischen Genreregimen, vor allem durch eine strukturelle Zentralisierung der Filmindustrie und somit eine staatspolitische Hierarchisierung der Genres. Zum Beispiel wurden in der UdSSR eine Zeit lang diejenigen Filme mehr anerkannt und daher besser finanziert, die sich stärker mit dem Schicksal des (sowjetischen) Kollektivs als dem der einzelnen Individuen beschäftigen. So wurde der Kriegsfilm nach 1945 zu einem solchen Staatsgenre, in dem sich sowjetische Identitäten nach 1945 formiert haben und aktualisiert wurden (vgl. GRADINARI 2020). Ähnlich verhält es sich mit dem DEFA-Antifaschismusfilm, der ostdeutsche Identität vor dem Hintergrund des Widerstandes gegen den Nationalsozialismus gestaltete. Dieses Genre ist jedoch nicht allein auf den Kriegsfilm begrenzt, sondern umfasste zum Beispiel auch den Gegenwartsfilm. In der Bundesrepublik haben die Kriegsfilme gelegentlich eine Konjunktur, konnten bis jetzt aber keine vergleichbare Bedeutung erlangen. Die Tatort-Filmreihe, die den Föderalismus der Republik und deren aktuelle soziale Probleme verhandelt, erhebt in den 1970er und 1980er Jahren den Anspruch, als Staatsgenre zu fungieren. Auch die Heimatfilme der 1950er Jahre können als ein solches Staatsgenre in jener Periode betrachtet werden, beantworten die Werke doch mit Entwürfen einer in dieser Form nie existierten Heimat politische Bedürfnisse der Zeit (vgl. TRIMBORN 1998:33). So möchte ich das Staatsgenre als eine Meta-Definition verstehen, zu der potenziell jede Filmgattung 
aufsteigen könnte, sobald diese als kollektiv und national wertvoll diskursiviert wird.

Der barocke Staatsroman ist in diesem Zusammenhang insofern interessant, als dass er eines der frühesten Beispiele der politischen Genres darstellt, das die Formierung des modernen Staates reflektiert und bedient. Der Staatsroman war in der Regel eine Utopie, bei der ein Staatsideal im Kontext einer Reise oder ausgehend von einem Dokumentennachlass aus einer idealen Welt entworfen wurde. Eines der früheren Beispiele stellt Utopia (1516) von Thomas Morus dar (zu verschiedenen Formen vgl. REICHERT 1965; JEßING 2007; JORDHEIM 2007). Nach einer der früheren Definitionen dieses Genres handelt es laut Johann Christoph Gottsched vor allem von Helden und fürstlichen Personen, die in einer erhabenen Form, „in einer edlen Schreibart“, erscheinen (GOTTSCHED 1962:154). Helge Jordheim verbindet diese Gattung mit der Herrschaftsform des Absolutismus, da der Staatsroman die Grenzen zwischen der Literatur und dem Fürstenspiegel verwischt und sich an die herrschende Elite, den höfischen Adel, wendet (vgl. JORDHEIM 2007:6). In diesem Zusammenhang definiert er den Staatsroman als jene Form, die ,politische und poetologische Fragestellungen“" narrativ verknüpft und entfaltet (JORDHEIM 2007:6). Die zentralen Topoi des Staatsgenres, an denen entlang auch die Narration entwickelt wird, sind nach Jordheim „der verborgene Prinz“, der „,redliche Mann am Hofe“, die „Fürstenerziehung“, die „Hofkritik“, der „Geheimbund“ und die „Staatsaktion“ (JORDHEIM 2007:6). Der Staatsroman ist nicht nur in Bezug auf seinen Inhalt politisch, sondern vor allem durch seine didaktischen Implikationen (JORDHEIM 2007:11). Von all den Merkmalen scheinen einige noch heute von zentraler Bedeutung zu sein: Der Staatsroman dient erstens als Stabilisierung des Staates, indem seine (bestehenden) Formen weitergedacht werden und so aus der Zukunft heraus legitimiert werden. Zweitens scheint dieses Genre eine andere Wirkung zu haben als andere Genres, nämlich theoretisch-philosophische Konzeptionen in seine Gestaltung zu integrieren und in seiner erzieherischen Dimension zugleich als eine Anleitung zur Handlung zu fungieren. Es überschreitet ästhetisch den Rahmen des Romans und nimmt sich dadurch das Recht heraus, sozusagen in das ,Leben“ hineinzugreifen, wenngleich es durchaus auch eine Unterhaltungsqualität haben kann. Drittens sind seine Figuren mit der Macht verknüpft - sie handeln also als Machtsubjekte, die durch ihre gesellschaftliche Position eine gemeinschaftliche Bedeutung haben und mit ihren Handlungen auf die Gesellschaft einwirken können. 
Das Kino als Massenkunst wurde bereits früh als machtvolles didaktisches und politisches Medium erkannt, wenn man zum Beispiel an Eisensteins intellektuelle Montage im Hinblick auf die Erschaffung neuer Menschen bis hin zur Reflexion neuer, durch den Film unwiderruflich veränderter Denk- und Wahrnehmungsprozesse denkt, die zunächst Béla Balász und dann Gilles Deleuze beschrieben hat. Spätestens seit dem Zweiten Weltkrieg wird die politische Dimension des Kinos anerkannt (vgl. ELSAESSER 1999). Es wird als Propaganda- und Aufklärungsmedium verwendet, vor allem aber als proto-dokumentarisches, ja mimetisches Medium verstanden, das imstande ist, ,Realität' ein Stück festzuhalten, also etwa als kollektives Speicher- und Erinnerungsmedium zu fungieren.

Für Hollywood ist das Konzept des Staatsgenres eventuell doch ,totalitär', so konnten einzelne Produktionen als Staatsfilme identifiziert werden, wenn sie ein großes Budget bekamen und es ihnen dabei geglückt ist, erfolgreich an aktuelle Fragen der Gegenwart anzuknüpfen und auf diese Weise eine große politische Wirkung oder zumindest öffentliche Resonanz zu erzielen. Daher können einzelne Western, Biopics und historische Dramen als Staatsfilme angesehen werden, wenn sie der Staatsmythologie (,Ursprung' des Staates, wichtige politische und historische Ereignisse, Reflexion der Staatsstrukturen usw.) zuarbeiten. Dazu können der rassistische Film The Birth of a Nation (USA 1915, R.: D.W. Griffith) und aktuelle kritische Antworten darauf wie The Birth of a Nation (USA 2016, R.: Nate Parker), dann JFK (USA 1991, R.: Oliver Stone), Malcolm X (USA 1992, R.: Spike Lee), Schindlers List (USA 1993, R.: Steven Spielberg), Django Unchained (USA 2012, R.: Quentin Tarantino), Lincoln (USA 2012, R.: Steven Spielberg) oder The Post (USA 2017, R.: Steven Spielberg) gezählt werden, um einige wenige Werke als potenzielle Staatsfilme zu nennen. Dabei erweist es sich in Hollywood als aufschlussreich, einzelne Regisseur*innen zu identifizieren, die einen besonderen erhabenen Drehstil entwickelten, sodass sie von Produktionsfirmen mit großem Budget unterstützt werden. Auffällig häufig gelangen Filme unter der Regie von Steven Spielberg zu einer breiten öffentlichen Resonanz. Auch im Film Saving Private Ryan, den ich ebenfalls als Staatsfilm betrachte, übernahm der bewährte Meister der historischen Epen Regie. Mit einem Budget von 70 Millionen Dollar spielte Saving Private Ryan bereits am ersten Wochenende in den USA über 30 Millionen Dollar ein. Insgesamt erzielten die Kinokassen in den USA Einnahmen von ca. 217 Millionen Dollar und international insgesamt über 480 Millionen Dollar mit diesem Film. Er bekam viele Auszeichnungen, darunter fünf Oscars. Die Einnahmen wie die Preise zeigen seine politische Relevanz, die auch auf das Thema des Films zurückzuführen ist: Saving Private Ryan handelt von einem 
internationalen, historisch und politisch relevanten Ereignis im Zweiten Weltkrieg, der Landung der US-amerikanischen Soldaten in der Normandie. Die USA wird als eine Weltmacht inszeniert, die internationale Geschichte beeinflusst. Die Besonderheit der Hollywood-Staatsfilms liegt dabei auch in der Häufung der Stars in einem Werk; allein die Besetzung unterstreicht somit die Relevanz des Themas. In den Hauptrollen spielen der bereits weltweit berühmte Tom Hanks und der damals noch junge Matt Damon. Auch viele Schauspieler in den Nebenrollen waren teilweise bekannt oder Spielbergs Film hat ihnen zum Aufstieg verholfen. Spielbergs Filme versammeln in der Regel zahlreiche Stars, um die persuasive Kraft des Kinos zu entfalten. Denn die Ansammlung von Stars überschreitet die ökonomische Logik der etablierten HollywoodProduktion - berühmte Schauspieler*innen übernehmen hier Rollen gerade wegen der besonderen Bedeutung des Filmthemas. So wird der Film zum Ereignis, bei dem die Stars performativ die Historie beleben und diese in Vertretung fürs Publikum erleben, was einen besonderen Wahrheitsgehalt erzeugt. Auch wenn es sich historisch nicht so ereignet hat, wie die Filme es durchspielen, haben zumindest die Schauspieler*innen es miterlebt und als Stars für Zuschauende bezeugt.

Der Film hat also das Staatsgenre neu definiert: Im Gegensatz zum elitären, intellektuell-philosophischen, barocken Staatsroman, gehören Filme zum Staatsgenre oder werden zu Staatsfilmen, indem sie sich gerade nicht auf ein elitäres Publikum oder eine elitäre Klasse beschränken. Die Filme zielen auf eine massenhafte Rezeption ab - über soziale Differenzen hinweg. Um das Publikum anzuziehen, wird der Film mit einem großen Budget produziert, um historische Authentizität und somit die Qualität des Kinoerlebnisses zu erhöhen. Auch viele Stars fördern eine breite Rezeption und einen Bedeutungszuwachs, die dann an der Kasse und ferner an Auszeichnungen abzulesen sind. In ihrer großen Wirkung überschreiten die Staatsfilme ihre ästhetischen Dimensionen und ihre geschichtliche Fiktionalität - an dieser Stelle ähneln sie dem Staatsroman - da sie oft auch als proto-dokumentarisch und ,wahrhaft' rezipiert werden. Diese Filme behandeln dabei den ,Ursprung' des Staates oder zumindest einen historischen Aspekt des Staates, der durch eine in der Regel als episch gestaltete Inszenierung in seiner Bedeutsamkeit (bis hin zum Ursprungsereignis) angehoben wird. Hat der Staat eine Biografie und somit das Kollektiv eine gemeinsame Geschichte, so wird die Nation nicht mehr in Bezug auf die Zukunft, sondern aus der Vergangenheit heraus legitimiert, weil eben nun die Vergangenheit als audiovisuelles Dokument präsent und evident ist und so eine starke Beweiskraft resp. Rechtfertigung der Existenz des Staates liefern kann. Eine 
Traditionslinie in der Vergangenheit zu stiften, legitimiert die Gegenwart, da die bestehende Staatsform nun aus der historischen Kontinuität heraus als eine bewährte und entwicklungslogische erscheint. Es geht also nicht mehr um konzeptionelle, symbolisch-logische Reflexionen zum Staatswesen, wie es im barocken Staatsroman der Fall war, sondern um affektive und emotionale Ereignisse, wobei beide doch teleologisch ausgerichtet sind - auf das zukünftige Bestehen des Staates. Weiterhin geht es dabei nicht mehr um einen Idealstaat, sondern um eine Krisenbewältigung. Der Staat wird einer Bewährungsprobe unterzogen und besteht diese erfolgreich, wodurch die aktuelle Ideologie oder politische Agenda den Opfern einen Sinn verleiht und so umgekehrt der Staat, für den die Opfer erbracht wurden, gestärkt wird.

Da es um Filmbilder geht, werden allerdings Gemeinschaften womöglich nicht mehr primär durch die Sprache konstituiert und nicht als begrenzt und souverän vorgestellt, wie es Anderson einst begründete, und vielleicht geht es gar nicht mehr um nationale Gemeinschaften überhaupt, sondern eher um einen Anschluss der Individuen an eine Geschichte und somit an die Macht imaginierter Traditionen, an ein Politisches-Imaginäres (vgl. CASTORIADIAS 1990; DOLL / KOHNS 2014). Dieser Anschluss wird durch eine affektive Bildlichkeit produziert, und genau deshalb bleibt dieser Prozess symbolisch offen. Die Bilder sind auf Erleben und Mitfühlen, nicht unbedingt auf einen Sinn ausgerichtet. Der Film ermöglicht so die Partizipation an einer anderen politischen Kollektivform bzw. führt er dazu, dass diese als Ereignis emotional erlebbar wird. Ein nationales Bewusstsein wird daher nicht mehr auf ein Territorium oder bestimmte Traditionen begrenzt, sondern erscheint als generisches Bilderrepertoire, auf das auch die reale Politik zurückgreift. Das funktioniert nur insofern, als dass wir die Geschichte eines Staates, einer Nation oder einer Gemeinschaft als eine für uns zur Identifikation angebotene individuelle Geschichte akzeptieren, wofür der Film Figuren allegorisiert. Die Allegorisierung entsteht aus der Verbindung der Figuren mit dem Kollektiv, das der Figur intradiegetisch historische und politische Macht verleiht - die Figur macht so eine global wirkende Geschichte und bestimmt die historische Entwicklung des gezeichneten Kollektivs. Es geht also um die Historie einer (oft nicht weniger imaginären) Gemeinschaft, zu deren Vertreter*innen die Zuschauenden werden sollen und die über filmische Strukturen international vermarktbar wird. 


\section{Allegorien und Position der Zuschauenden}

Die Allegorie ist eine Stilfigur der uneigentlichen, metaphorischen Rede, die im Falle der Personifikationen in einer Figur zum Ausdruck kommt, welche auf eine andere Bedeutung verweist. Als gängiges Beispiel dient Justitia. Die lange Tradition der Nationalallegorien stammt aus der Kunstgeschichte. In der Regel handelt es sich um Frauenfiguren, wie die feministische Forschung überzeugend ausgearbeitet hat (vgl. WAGNER 1989). Männliche Figuren präsentieren oft reale historische Persönlichkeiten; sie treten als historisch und politisch handlungsfähige Subjekte auf. Weibliche Allegorien besitzen dagegen nach Aleida Assmann keinen individuellen Charakter und keine Geschichte (vgl. ASSMANN 1994:25). Das heißt, dass die Nation über das Andere - eine idealisierte, reine, in der Regel jungfräuliche Weiblichkeit - als Natürliches, zugleich für das männliche handelnde historische Subjekt verfügbar und seinem Willen unterworfen inszeniert wird.

Als Vorläuferin der filmischen Allegorien könnte u.a. die Historienmalerei des 19. Jahrhunderts verstanden werden, da sie eine empathische Involvierung der Betrachtenden in das historische Geschehen zu erzielen suchte, was eigentlich schon als profilmisches Verfahren beschrieben werden kann. Der Kunsthistoriker HEINZ-TONI WAPPENSCHMIDT (1984:61) hat darauf hingewiesen, dass im ausgehenden 19. Jahrhundert die Wände einiger Rathaussäle, zum Beispiel in München, Worms, Hildesheim und Bochum, mit historischen Ereignissen und mit weiblichen Allegorien bemalt wurden. Die Allegorien an den Wänden wurden derart nach Wappenschmidt in die Struktur des Versammlungsraums integriert, sodass in der Regel der Vorsitzende vor der Wand mit Allegorien im Rücken saß und so beide Zeiten verbunden wurden - die mythische Zeit der Bilder und die Zeit politischer Entscheidungen in der Gegenwart (Abb. 1-2). 


\section{Irina Gradinari}

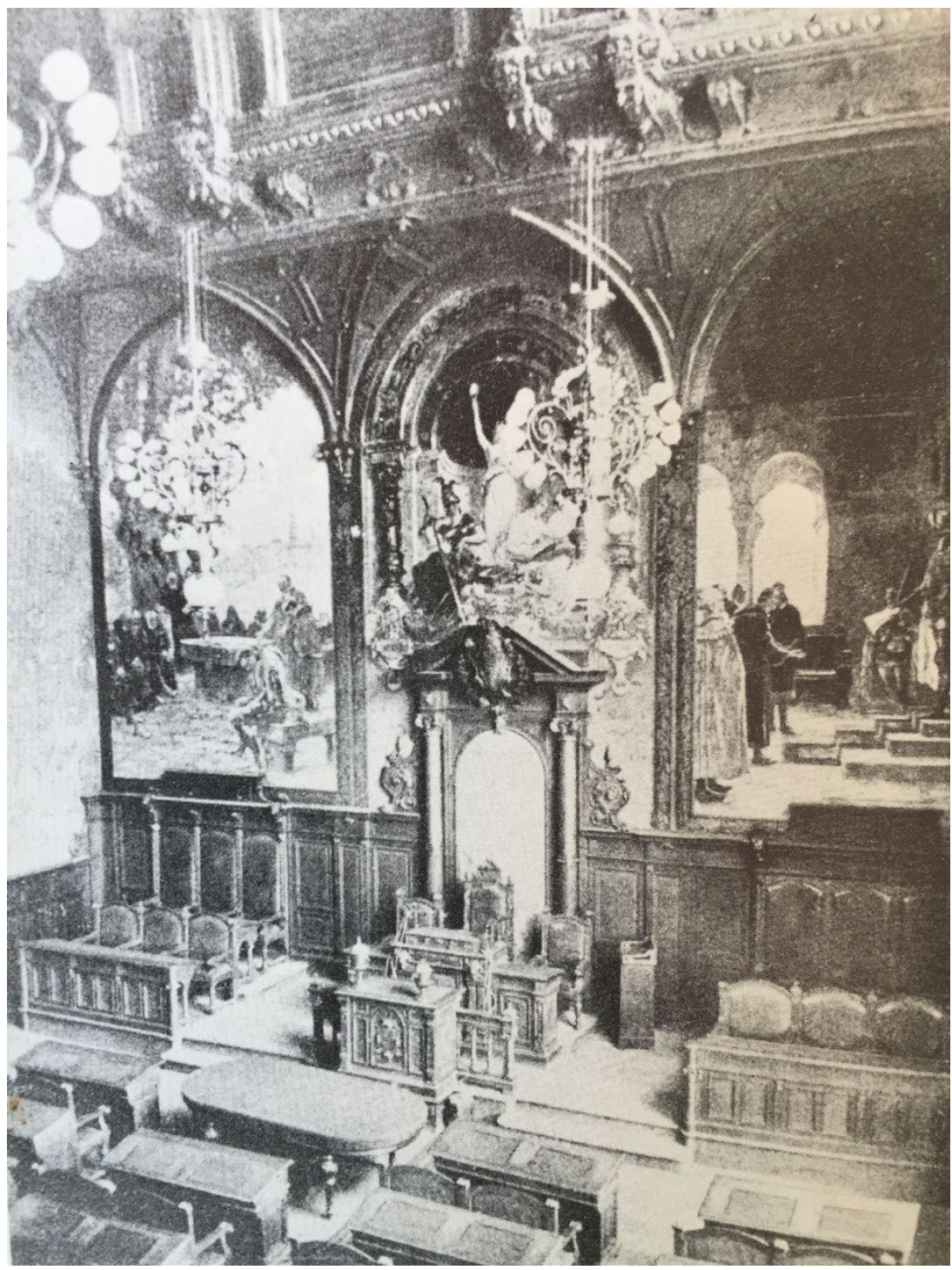

Abb. 1: Die Nordwand des Bochumer Rathaussaales: Von links nach rechts sind das ,Feme-Gericht', ,Kraft - Wahrheit - Recht' und die ,Verleihung des Stadtrechts' dargestellt, von Fritz Neuhaus aus dem Jahr 1898/1901 (vgl. WAPPENSCHMIDT 1984:71-75). 


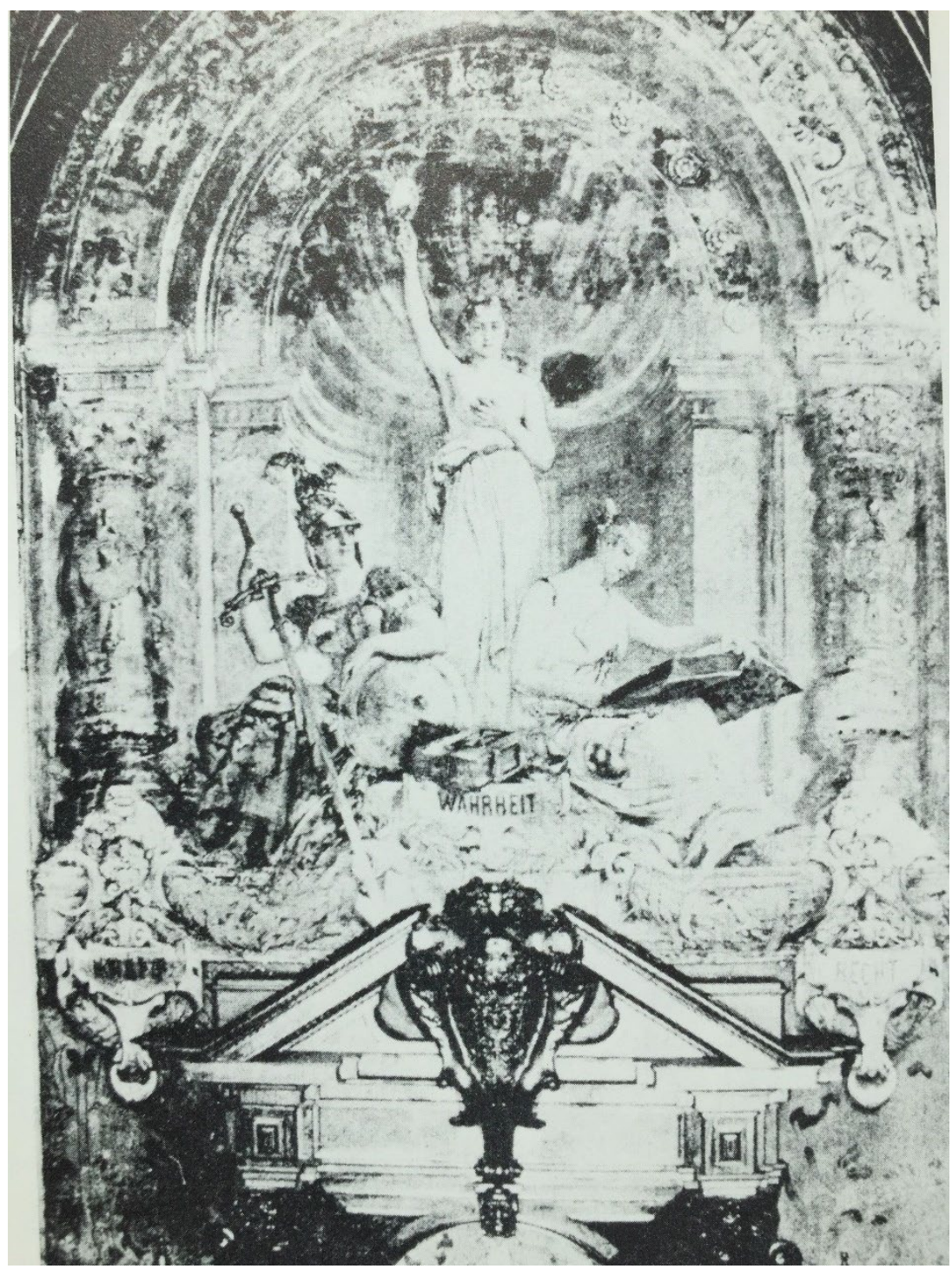

Abb. 2: In der Mitte über und hinter dem Vorsitzenden befinden sich die weiblichen Allegorien: Links sitzt die Allegorie Kraft mit einem Schwert, in der Mitte steht die Allegorie Wahrheit mit einer Fackel in der Hand und rechts sitzt die Allegorie Recht mit einem Buch (vgl. WAPPENSCHMIDT 1984:71-75). 
Der Vorsitzende wurde auf diese Weise selbst zum Bestandteil der historischen Bilder und vor diesem Hintergrund zur Allegorie der Gegenwart, zugleich sollten mythische Bildallegorien seine Entscheidungen legitimieren. Zusammen wurden sie zu einer visuellen Einheit, die als politische Praxis identifizierbar ist und die weiterhin in der bildlichen und damit filmischen Propaganda tradiert wurde (vgl. WAPPENSCHMIDT 1984:71-75). Damit wird schon hier deutlich, dass auch Männer durch die Architektur des Raums allegorisiert werden können.

Der Film veränderte Allegorien weitgehender. Aufgrund der Blicklenkung und der narrativen und ästhetischen Strukturen, die die Zuschauenden dazu einladen, sich mit der Hauptfigur zu identifizieren, werden Allegorien im Film nicht oder zumindest nicht ausschließlich über das Andere produziert, sondern über die Hauptfigur. So sind zum einen im Film zahlreiche männliche Allegorien zu finden, da in historischen und biografischen Filmen vor allem männliche Hauptfiguren vorkommen. Die Produktion von Allegorien im Film ist zum anderen stärker an die Rezeption gebunden - die Zuschauenden werden gemeinsam mit der Hauptfigur performativ zur Allegorie. Das wird insofern möglich, als dass sich die Zuschauenden narrativ dazu ,verführen“ lassen bzw. diese Identifikation, darauf verweist STEPHEN HEATH (vgl. 1981:53), aktiv angenommen werden muss: Die Zuschauenden müssen sich darauf einlassen, in die filmischen Bilder und Bewegungen einbezogen zu werden. Identifikatorische Strukturen funktionieren dabei nicht geschlechtsanalog, sonst wäre eine breite Rezeption der Genrefilme nicht möglich. Das liegt nach TERESA DE LAURETIS (vgl. 1990) daran, dass die Involvierung der Zuschauenden über zwei Figuren erfolgt, über die „Figur der Bewegung“, also das Subjekt der Erzählung, und die „Figur ihres Abschlusses“, das abschließende „Erzählbild“ (narrative closure), in dessen Richtung sich die Narration entwickelt und das die Handlung zu einem Ende bringt:

Beide sind figürliche Identifikationen, beide sind gleichzeitig möglich; mehr noch: sie werden gleichzeitig vom narrativen Prozeß hervorgebracht und wechselseitig miteinander verwoben. Diese Art der Identifikation würde beide Positionalitäten des Begehrens, sowohl aktive als auch passive Strebungen tragen: das Begehren nach dem Anderen, und ein Begehren, von anderen begehrt zu werden. (LAURETIS 1990:13)

De Lauretis zeigt damit, dass die Frauenfiguren sowohl als Objekte des Blickes in Identifikationsprozesse involviert werden als auch die Position des Subjektes besetzen können. Das kann auch für männliche oder queere Figuren behauptet werden, sobald mit ihnen historische Ereignisse inszeniert werden. Generell ist die sozialhistorische, typisierende oder allegorische Codierung der Figur im Film aufgrund seiner spezifischen Eigenschaft, Figuren durch das Mise en 
Scène zu semantisieren, kaum zu vermeiden (vgl. RITZER 2017). In diesem Zusammenhang bewirkt die generische Darstellungspraxis jene Abstrahierung der Figuren vom Individuellen ins Allgemeine, sodass sie auf diese Weise zur Vertretung des Kollektivs werden.

Der Allegorisierung geht zunächst eine Typisierung voraus: Im ersten Schritt bedeutet dies eine Abstrahierung der Figur vom individuellen Kontext. Im zweiten Schritt, bei dem Erlangen des Abschlussbildes, wird die Figur dann durch deren Abstrahierung aus einem konkreten historischen Kontext allegorisiert, was ja gerade eine für die Allegorie typische Zeittranszendenz bedeutet. Um die Allegorisierung der Figuren zu erreichen, werden sie also zunächst in kollektiven Handlungsbelangen oder kollektivrelevanten Kontexten situiert, indem sie an öffentlichen Schauplätzen auftreten und von staatsrelevanten Symbolen und historischen Zitaten bekannter Personen begleitet werden. In der Regel nehmen die Figuren dabei an erinnerungsrelevanten Ereignissen teil. Dies alles bewirkt, dass Figuren, ihre Identität und Begehren, als exzentrisch - räumlich außerhalb ihrer selbst - produziert werden, was ihre typisierende Abstraktion bewirkt. Im nächsten Schritt vollzieht die Figur eine Verwandlung zu einer Allegorie, indem sie in der Nahaufnahme erstarrt und so körperlos und in der Regel auch als unsterblich - zum Beispiel vor dem Hintergrund des Himmels - erscheint, da das Leben des Kollektivs nicht mit dem individuellen Tod endet. Bildlich wird eine transgenerationelle Kontinuität erschaffen, womit die Allegorie als zeitlose Figur hervortritt.

Allegorien im Film unterliegen daher einem dynamischen Prozess: Die Zuschauenden können sich mit dem zunächst individualisierten Subjekt der Diegese identifizieren und sich auf die exzentrische Entwicklung durch die Diegese einlassen. Durch diese Entwicklung vollziehen sie weiterhin gemeinsam mit den Figuren performativ die Verwandlung in ein Allegoriebild, das in der Regel gegen Ende der Handlung hervorgebracht wird und so zugleich mit dem narrativen Abschlussbild zusammenfällt. Das abschließende Erzählbild wird als durch die Erzählbewegung installierter Sehnsuchtsort gegen Ende aufgesucht.

\section{(Inter-)Nationale Allegorie in Saving Private Ryan}

Bereits generisch ist der Kriegsfilm dafür prädestiniert, Nation und Staat zu thematisieren, arbeitet er doch an der Bildung eines Kollektivs bzw. einer Männergruppe. In der Gruppe verdichten sich spezifisch männliche Konflikte wie unterschiedliche Hierarchieaushandlungen, Kameradschaft und Ehre oder auch 
nationalspezifische Themen, etwa der solidarische Zusammenschluss gegenüber der Gefahr und das Erbringen eines Opfers für die Heimat (vgl. KLEIN / STIGLEGGER / TRABER 2006:17-20). All diese Motive finden sich in Saving Private Ryan. Zwei Figuren, Captain John H. Miller (Tom Hanks) und James Ryan (Matt Damon), werden dabei hervorgehoben, sie wechseln sich in der Rahmen- und Binnenhandlung ab. Mit ihnen wird jene narrative Bewegung inszeniert, die sich aus dem Jetzt in die Vergangenheit bewegt, um wieder zurück in die Gegenwart zu gelangen. Die Figur der Erzählbewegung stellt Captain Miller dar; das Abschlussbild findet in James Ryan seinen Ausdruck.

Die Zeitreise in die Vergangenheit und eine Reise nach Frankreich sind zudem durch die Such- und Rettungsaktion intradiegetisch motiviert - James Ryan ist der vierte und letzte noch lebende Sohn der Familie Ryan, der nun gefunden und nach Hause geschickt werden soll. Seine drei älteren Brüder sind kurz zuvor gefallen, womit die Opfer des US-Volkes im Zweiten Weltkrieg zunächst in der international tradierten Ikonografie angedeutet werden. Das Motiv der trauernden Mutter-Heimat um ihre gefallenen Söhne ist in den sowjetischen, ost- und westdeutschen Kriegsfilmen zu finden (vgl. GRADINARI 2020:114f.). Die Narration fügt sich mit der Landung der Alliierten zu Beginn des Filmes in kollektive historische Zusammenhänge ein, zugleich widersetzt sich die Rettung eines einzigen Soldaten der historischen wie auch der Kriegslogik - es handelt sich um das aus der Gegenwart heraus artikulierte Begehren, einzelne Akteure $\mathrm{zu}$ retten und $\mathrm{zu}$ memorieren, das Wissen über die eigene Historie nicht zu verlieren.

Diese Handlungsentwicklung bedeutet zudem, sich von der privaten zur gesellschaftlich relevanten Sphäre zu bewegen, von der individuellen zur historischen Wirkungsdimension aufzusteigen. Zu Beginn des Films ist eine US-amerikanische Familie zu sehen. Vor allem der Vater, wahrscheinlich ein Kriegsveteran, spielt eine große Rolle. Die Handlung startet auf dem berühmten amerikanischen Soldatenfriedhof, Normandy American Cemetry, in Colleville-sur-Mer in Frankreich, wodurch die Richtung der narrativen Reise bereits angedeutet wird. Es geht also um den Zweiten Weltkrieg, die Landung der Alliierten in der Normandie, die als Erinnerungsort nicht allein auf eine Nation bezogen werden kann. Dieses Ereignis gehört zur deutschen, französischen und USamerikanischen Kriegsikonografie. So ist die Historie von Anfang an doppelt inszeniert - als eine US-amerikanische Geschichte, die jedoch zu einem gemeinsamen internationalen Erinnerungsbild über nationale und zeitliche Grenzen hinweg werden soll. Auch in der Binnenhandlung werden weitere kollektive und zugleich im Kriegsfilm tradierte Schauplätze aufgesucht und Schlachten 
ausgetragen, zum Beispiel die Verteidigung einer Brücke. Kollektive historische Schauplätze lassen Figuren zu Teilnehmenden, somit zu Zeugen historischer Geschehnisse werden und heben zugleich ihre gemeinschaftliche Bedeutung hervor.

Die Familie wird in diesem Kontext zum Sinnbild der US-Nation, wobei ihr metaphorischer Charakter erst gegen Ende des Films zur Geltung kommt, wenn wieder die Gegenwart gezeigt wird. Zu Beginn wissen wir noch nicht, was es mit der Familie auf sich hat, wobei das Ambiente symbolisch stark aufgeladen ist, was eine der wichtigen Strategien darstellt, Allegorisierung zu produzieren: Zahlreiche Kreuze, die auf das kollektive Opfer hinweisen, Flaggen, die US-amerikanischen Stars and Stripes und die französische Tricolore als Staatssymbole, die hervorheben, für wen das Opfer erbracht wurde, eine große Familie - ein eigentlich protestantisches Ideal des verwirklichten Lebens - und ein musikalisches Motiv, bei dem das Horn hervorsticht. Blasinstrumente, die bei feierlichen Anlässen und in Militärmärschen häufig verwendet werden, wurden auch im sowjetischen, west- und ostdeutschen Film eingesetzt, um die Erhabenheit des Bildes zu unterstreichen und so Pathos zu produzieren. Außerdem wird der Klang der Trompete oder hier des Horns als Ruf des Kollektivs und der Vorfahren inszeniert, in deren Schuld die Figuren als Überlebende und somit auch die Nachkommen stehen. Die Familie wird in diesem Kontext zum Sinnbild der US-Nation, das die Zuschauenden zu vervollständigen berufen sind (vgl. KAPPELHOFF 2016). Durch Annäherung der Kamera bildet die Familie einen Halbkreis, den das Publikum seitens der Leinwand ergänzt, indem es mit den Figuren einen in den Kinosaal hineinreichenden Kreis bildet (Abb. 3).

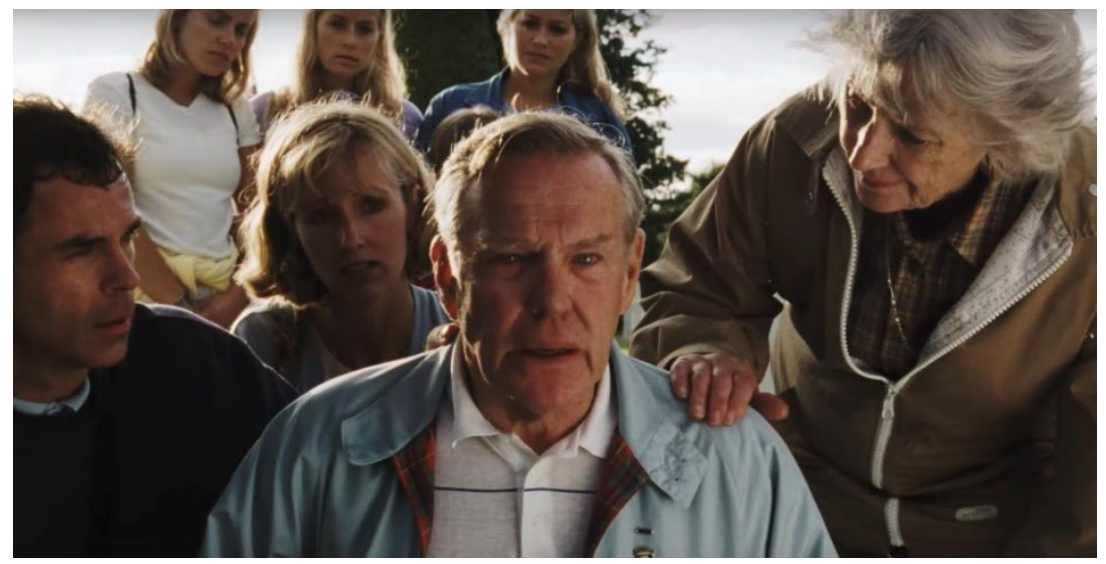

Abb. 3: Saving Private Ryan. Die Einstellung leitet zur Binnenhandlung über. 
So wird bereits zu Beginn jenes Bild der Nation aufgerufen, das sich auf eine Kontinuität zwischen Vergangenheit und Gegenwart beruft: Die Figur aus der Rahmenerzählung gehört der Geschichte und zugleich der Gegenwart an, sie fungiert als Brücke in die Vergangenheit und Zeitzeugin, die das Geschehen authentifiziert. Die Figur bereist stellvertretend für die Zuschauenden die Geschichte und begründet aus der Vergangenheit heraus die Gegenwart als eine gerechte und ruhmvolle Ordnung, hat doch der Überlebende in Erinnerung an die Opfer dem Staat gedient und so zu seinem Gedeihen - die Nachkommenschaft ist dafür ein Beweis - verholfen. Um diese Figur zu typisieren, wird die Filmhandlung zudem so inszeniert, dass letztendlich jede*r die Nation vertreten kann: Die Figur in der Rahmenhandlung bleibt anonym. Bis zum Filmende wissen wir nicht, welche der beiden Figuren, Captain Miller oder James Ryan, überlebt hat. Der Schnitt, der die Rahmenhandlung der Gegenwart und die historische Landung in der Normandie in der Binnenhandlung verbindet, fügt die gegenwärtige Figur mit Captain Miller im Boot zusammen, sodass wir dazu verleitet sind, zu denken, der alte Mann in der Rahmenhandlung sei diese Figur, die von Tom Hanks gespielt wird. Diese Zusammenführung suggeriert, dass individuelle Erinnerungen der Figur in der Rahmenhandlung rekonstruiert werden. Erst gegen Ende des Films erfahren wir, dass der alte Mann ein anderer, eben der gerettete Soldat Ryan ist, der sich also gar nicht auf diese Weise an die gezeichnete Landung in der Normandie erinnern konnte. Der Ersatz einer Figur durch die andere bildet eine Metapher für das Weiterleben des Kollektivs und authentifiziert nachträglich die gezeigten Schlachten als historische Dokumente, die allumfassend die Geschichte archivieren und individuelle Erinnerungen weit überschreiten. Solche Strategien entkoppeln regelrecht das historische Geschehen von subjektiven Empfindungen, objektivieren diese als eine das individuelle (Erinnerungs-)Vermögen übersteigende historische ,Wahrheit' (so ist es eben gewesen) und signalisieren wiederholt, dass es nicht um ein Individuum geht, sondern um das Kollektiv oder eine Nation, zu deren Vertretung in diesem Fall jeder Soldat werden könnte. Die Figur wird als exzentrisch produziert - außerhalb ihres familiären Kontextes, sogar außerhalb der eigenen Heimat.

Der letzte Schritt ist die Allegorisierungsszene selbst, in der der Soldat James Ryan durch den symbolisch überladenen Kontext, durch die Größe des erbrachten Opfers, die Betrachtung des Todes und die visuelle Abhebung der Figur in den Himmel in eine Allegorie der Nation verwandelt wird. Die Größe des Opfers wird sowohl narrativ produziert - die verstorbene Figur war die eigentliche Hauptfigur - als auch durch das Image des Stars Tom Hanks, der 
entsprechend der Hollywood-Logik gar nicht sterben darf: Die von Matt Damon gespielte Figur überlebt und wird im Angesicht des Todes bildlich und inszenatorisch auf eine andere Ebene gehoben. Sie wird von unten gefilmt, wodurch sie in den Himmel entrückt wird (Abb. 4-5).

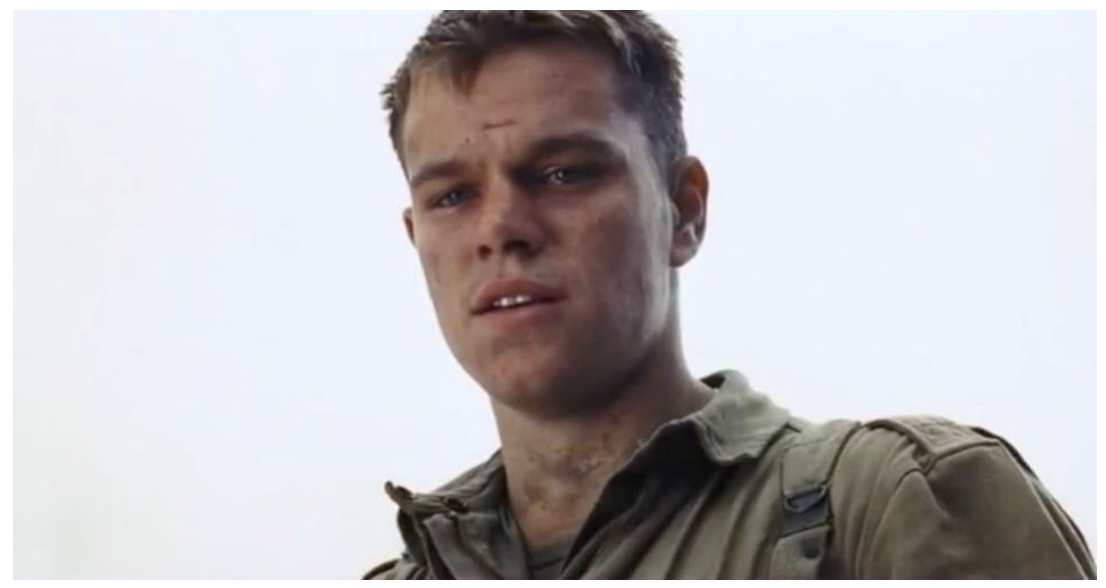

Abb. 4: Der Soldat James Ryan ist beim Betrachten des gefallenen Captain Miller zu sehen.

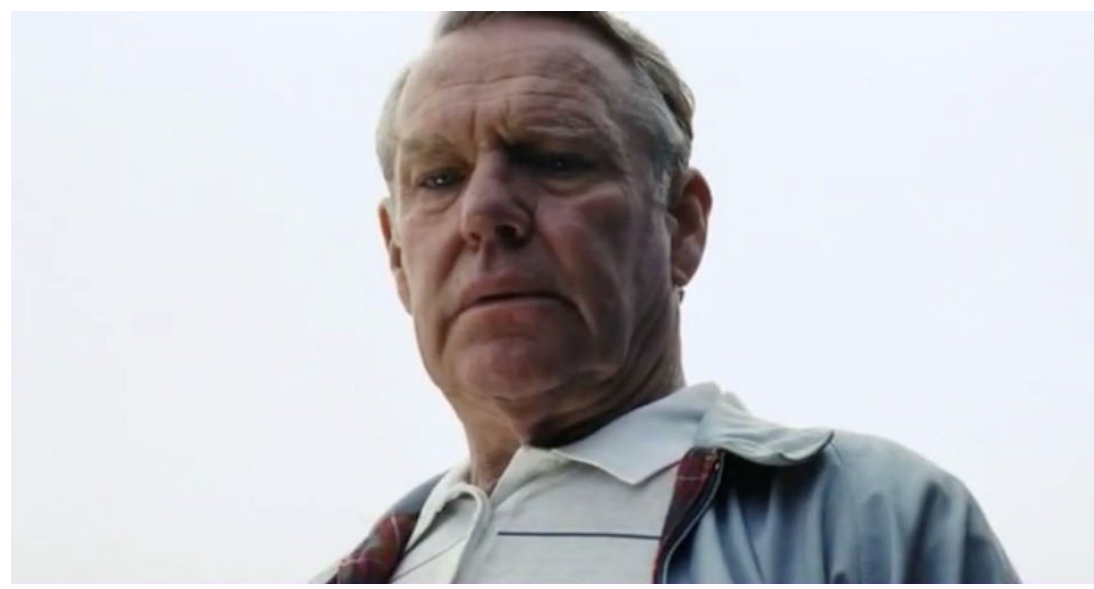

Abb. 5: Durch den Morphing-Effekt altert das junge Gesicht, bis es zum Gesicht der Figur aus der Rahmenhandlung auf dem Soldatenfriedhof wird, wodurch der Zeitsprung zustande kommt. 
So ist einerseits jede*r, der*die bereit ist, das eigene Leben für andere zu opfern, ein Bestandteil der Nation. Andererseits endet die Nation nicht mit dem Tod eines Individuums, wodurch die Figur einer anderen Zeit oder vor dem Hintergrund des Himmels sogar der Ewigkeit angehört und dadurch in eine Allegorie der Nation verwandelt wird. In der Regel erstarren die Figuren im Moment ihrer Allegorisierung, was visuell ihre Monumentalität hervorhebt. Dadurch fällt es in einem dynamischen Medium wie dem Film natürlich besonders auf, wenn die Figur letztlich zum Bild wird. Diese Erstarrung signalisiert eine andere Zeitlichkeit, die für die Allegorie nach Paul de Man von zentraler Bedeutung ist: „In der Welt der Allegorie [...] ist die Zeit die ursprünglich konstitutive Kategorie. Die Beziehung zwischen dem allegorischen Zeichen und seiner Bedeutung (signifié) ist nicht dogmatisch festgeschrieben“"(DE MAN 1993:103). Der Verweis auf ein anderes Zeichen, das die Allegorie konstituiert, erzeugt die Nicht-Identität, die Distanz zum eigenen Ursprung, in der, so de Man weiter, „die Leere der zeitlichen Differenz“ (DE MAN 1993:104) sichtbar wird. In der besprochenen Filmsequenz von Saving Private Ryan wird die MorphingTechnologie verwendet, wodurch die Bewegung in der Zeit durch das Altern des Gesichts hervorgehoben wird. Die Figur der Rahmenhandlung ist mit der Figur der Binnenhandlung nicht identisch, wird auch durch zwei Schauspieler dargestellt, was die zeitliche Differenz sichtbar macht. Die Zeittranszendenz, die die individuelle Lebensdauer und die einer Generation überschreitet und so die Unsterblichkeit des Kollektiven inszeniert, unterstützen darüber hinaus die Worte Lincolns, die im Moment des Zeitübergangs zitiert werden und so auch auf ein anderes Opfer im US-amerikanischen Bürgerkrieg verweisen. Eine dunkle, männlich klingende Stimme aus dem Off, deren Einsatz Autorität und Wahrheit der Historie ausstrahlt (vgl. SILVERMAN 2016), zitiert aus dem berühmten Brief Lincolns an Mrs. Bixby, deren fünf Söhne in den Reihen des Unionsheers gefallen waren (vgl. BURLINGAME 1995). So spricht Lincoln durch die Zeiten zu den Zuschauenden. Aus diesem Verweis entsteht die Allegorie der Nation als eine zeitliche Figur, die drei Zeiten (die Gegenwart, die des 2 . Weltkrieges und die des US-amerikanischen Bürgerkrieges) verbindet und die auf ihr vorausgehende Zeichen verweist, zugleich jedoch Distanz zu ihrem eignen Ursprung markiert. Das soldatische Opfer im Zweiten Weltkrieg wird zurück auf den Sezessionskrieg bezogen und somit mit dem politischen Ursprung der modernen US-Gesellschaft verbunden - immer schon mussten heroische Männer ihr Leben auf den ,Altar der Freiheit‘ legen (,a sacrifice upon the altar of freedom" - wie es im Film mit Lincoln heißt), um so die Geschichte als wiederholt erbrachtes Opfer zu inszenieren, dadurch eine ge- 
schichtliche Kontinuität der Nation zu ermöglichen und zugleich die Überlebenden zu der Form der Nation/des Staates zu verpflichten, die sie verteidigt haben. Berühmte Worte Lincolns, vor allem die Gettysburg Address, haben in Hollywood eine große Tradition, staatlich Interventionen zu legitimieren (vgl. PAUSE 2021:33-61).

\section{Allegorie und Affektbild}

Zur Allegorie wird hier nun also nicht mehr das Andere, sondern das Subjekt. Die Zuschauenden vollziehen im Film mit dem narrativen Abschlussbild eine Verwandlung mit, die die Figur in eine Idee transformiert und so von einem konkreten sozialen oder historischen Prozess abstrahiert. Dieser Prozess der Entkörperlichung der Figur und der Abstrahierung des Bildes von einem konkreten historischen Kontext rückt der Überlebende im Bild in die Nähe der Zuschauenden: Die Distanz zum Bild und zum Geschehen wird so verringert, wodurch das Publikum affiziert, also geprägt und beeinflusst wird, bevor es sinnlich und sinnhaft erfassen könnte. Das Bild ergreift die Zuschauenden (vgl. MASSUMI 2015:103, MASSUMI: 2010). An dieser Stelle fällt die affektive Wirkungsstrategie des Films mit dem Affektbild im Sinne von GILLES DELEUZE (1996:123) zusammen, indem die Narration, die Bewegung im Bild und somit auch der mobile soldatische Körper einfrieren: „Dieses Ensemble aus einer unbeweglich reflektierenden Einheit und dichten expressiven Bewegungen konstituiert den Affekt" (DELEUZE 1996:124). In der Großaufnahme sowie im Anhalten der Narration wird die Allegorisierung durch die Auflösung des Bildes von den semiotischen Bezügen der Handlung (vgl. KAPPELHOFF 1995:46) produziert, gerade weil der Himmel nicht nur religiöse Konnotationen aufruft, sondern zugleich auch semantisch leer erscheint. Im Affektbild wird so die „virtuelle Vielheit“ erlebbar, wie ANKE ZECHNER (2006:157) das nie zu verbalisierende und festzulegende Potenzial der Bilder in Auseinandersetzung mit Gilles Deleuze bezeichnet. Das Bild öffnet sich also gegenüber allen möglichen Referenzen und Sinnzugängen, die zugleich nicht fixierbar sind. Im Sinne von BRIAN MASSUMI (2010) setzen solche Bilder auch Affekte frei. Zum einen bewegen sich die Filme des Staatsgenres immer schon im Bereich des ,MehrAls` das nach ERIN MANNING (2010:21) gerade eine Erfahrung der Überschneidung von Aktuellem und Virtuellem sowie die Erfahrung der Kollektivität darstellt. In den Filmen werden die Narrative und die Zeit des Individuellen ständig überschritten bzw. Individuen in Bezug auf die Gemeinschaft umgeschrieben, ihre Identität als exzentrische, als eine außer ihrer selbst, konstituiert. So evo- 
zieren die Bilder den Exzess des Kollektiven, der Zuschauende ständig überwältigt. Diese Überwältigung - eine der wichtigen Affizierungseffekte nach BRIAN MASSUMI (vgl. 2008; MANNING 2010:18) - hat auch eine geschlechtsauflösende Qualität, weil sich zum anderen die Großaufnahmen bekanntlich vom Körperlichen ablösen (vgl. DeLEUZE 1996:124). In dem Moment der Allegorisierung verlieren Figuren ihren geschlechtsspezifischen Körper; sie werden so zugleich von den Zwängen der geschlechtlichen Semantisierungsprozessen und der konkreten sozialen und politischen Zugehörigkeit im Bild befreit.

In Worten von Gilles Deleuze, der Eisensteins Großaufnahmen analysiert, erschafft das Affektbild eine dividuelle Realität, „die unmittelbar eine unermeßliche kollektive Reflexion mit den je besonderen Empfindungen jedes Individuums vereinigt und endlich der Einheit von Potential und Qualität Ausdruck gibt“ (DELEUZE 1996:129). In diesem Zusammenhang werden Figuren zum Gesicht des Kollektiven und so zum Monument der Epoche, auch weil sie oft wie Monumente bis zur Brust im Bild erscheinen. Allegorien rücken die $\mathrm{Zu}-$ schauenden somit in eine ageschlechtliche oder geschlechtsirrelevante Position, wodurch die Figur zur Verkörperung der Nation und so zum Signifikanten bzw. zur Idee von Staatlichkeit, Gerechtigkeit oder Kollektivität, die in dieser Form nur im Kino möglich sind, gelangt. Vage könnte dieses Ergriffensein vom Publikum zum Teil als Pathos und Erhabenheitsgefühl verspürt werden, als Gefühl, Bestandteil von etwas Größerem zu sein (auch durch das Verweisen auf vorherige Zeichen), das sowohl jene Lust an der Teilhabe an der Geschichte oder durch die Zugehörigkeit zu einem Kollektiv als auch eine Ablehnung wegen eines ideologischen Vereinnahmens durch das Bild erzeugen kann. Der Allegorisierung und somit der Affizierung selbst können die Zuschauenden jedoch nicht entkommen.

\section{Literatur}

ANDERSON, BENEDICT (1996): Die Erfindung der Nation. Zur Karriere eines folgenreichen Konzepts. Frankfurt a.M. / New York.

Assmann, Aleida (1994): Die Wissende und die Weisheit - Gedanken zu einem ungleichen Paar. In: Schade, Sigrid / WAgner, MoniKa / Weigel, Sigrid (eds.): Allegorien und Geschlechterdifferenz. Köln / Weimar / Wien, 11-25.

Burlingame, Michael (1995): New Light on the Bixby Letter. In: Journal of the Abraham Lincoln Association 16:59-71.

CASTORIADIAS, CORNELIUS (1990): Gesellschaft als imaginäre Institution. Entwurf einer politischen Philosophie. Frankfurt a.M. 
De Man, PAul (1993): Die Ideologie des Ästhetischen. Frankfurt a.M.

DeleuZe, Gilles (1996): Das Bewegungsbild - Kino 1. Frankfurt a.M.

DoAne, MARY ANN (1985): Film und Maskerade: Zur Theorie des weiblichen Zuschauers. In: Frauen und Film 38:4-19.

Doll, Martin / Kohns, Oliver (eds.) (2014): Die Imaginäre Dimension der Politik. München.

ElsAesser, ThOMAs (1999): Das Weimarer Kino - aufgeklärt und doppelbödig. Berlin.

ElsAesser, ThOMAs (2002): ,Un train peut en cacher un autre. “ Geschichte, Gedächtnis und Medienöffentlichkeit. In: Montage/AV 11,1:11-25.

GAMPER, MichAel (2010): Ideologie und Gattung. In: ZYMNER, RÜDIGER (ed.): Handbuch Gattungstheorien. Stuttgart, 66-69.

GOTTSCHED, JOHANN CHRISTOPH (1962): Versuch einer critischen Dichtung. Unveränderter photomechanischer Nachdruck der 4., vermehrten Auflage 1751, Darmstadt.

GRADINARI, IRINA (2020): Kinematografie der Erinnerung. Bd. 1: Filme als kollektives Gedächtnis verstehen. Wiesbaden.

HeAth, STEPHEN (1981): Questions of Cinema. Bloomington.

JAMESON, FREDERIC (1988): Das politische Unbewußte. Literatur als Symbol sozialen Handelns. Reinbek bei Hamburg.

Jeßing, Benedikt (2007). Staatsroman. In: Budorf, Dieter / FASBEnder, Christoph / Moenighoff, BurkHARD (eds.): Metzler Lexikon Literatur: Begriffe und Definitionen. Stuttgart / Weimar, 727-728.

Jordheim, Helge (2007). Der Staatsroman im Werk Wielands und Jean Paul. Tübingen. KaES, Anton (1987): Deutschlandbilder: Die Wiederkehr der Geschichte als Film. München.

Kappelhoff, Hermann (1995): Der möblierte Mensch. Georg Wilhelm Pabst und die Utopie der Sachlichkeit. Ein poetologischer Versuch zum Weimar Autorenkino. Berlin. KAPPELHOFF, HeRmanN (2016): Genre und Gemeinsinn: Hollywood zwischen Krieg und Demokratie. Berlin.

Klein, Thomas / Stiglegger, Marcus / Traber, Bodo (2006): Einleitung. In: Dies. (eds.): Filmgenres: Kriegsfilm. Stuttgart, 9-28.

LAndsberg, Alison (2016): Engaging the Past: Mass Culture and the Production of the Historical Knowledge. Columbia.

LAURETIS, TERESA DE (1990): Ödipus interruptus. In: Frauen und Film 48: Väter + Töchter:5-29.

MANNING, ERIN (2010): Das Ereignis des Schreibens: Brian Massumi und die Politik des Affekts. In: MASSUMI, BRIAN (ed.): Ontomacht. Kunst, Affekt und das Ereignis des Politischen. Berlin, 7-24.

Massumi, Brian (2008): Perception Attack: Brief on War Time. In: Multitudes Volume 34/3:74-83. 
Massumi, Brian (2010): Ontomacht. Kunst, Affekt und das Ereignis des Politischen. Berlin.

Massumi, Brian (2015): Keywords for Affect. In: Ders.: The Power at the End of the Economy. Durham, 103-112.

MoINE, RAPHAËLle (2008): Cinema Genre. Malden, MA.

Mulvey, Laura (1994): Visuelle Lust und narratives Kino. In: Weissberg, LiLiane (ed.): Weiblichkeit als Maskerade. Frankfurt a.M., 48-64.

Pause, Johannes (2021): Die zwei Geister des Präsidenten. Lincoln-Mythos und politische Theologie im Hollywood-Kino während der Great Depression: GABRIEL OVER the White House (1933). In: Gradinari, Irina / Niehaus, Michael (eds.): Destabilisierung - Störung - Verunsicherung. Dortmund, 33-61.

REICHERT, KARL (1965): Utopie und Staatsroman. Ein Forschungsbericht. In: Deutsche Vierteljahrsschrift für Literaturwissenschaft und Geistesgeschichte 39, Nr. 2:259-287.

RITZER, Ivo (2017): Medialität der Mise-en-scène. Zur Archäologie telekinematischer Räume. Wiesbaden.

Rosenstone, Robert A. (1993): Like Writing History With Lightning: Historical Film/Historical Truth. In: Contention 2.3:191-204.

Silverman, KaJa (2016): Die weibliche Stimme ent-körpern. In: SEIER, ANDREA/ Peters, KATHRIN (eds.): Gender und Medien. Zürich / Berlin, 71-90.

Sobchack, Vivian (1996): Introduction: History Happens. In: DiES. (ed.): The Persistence of History. Cinema, Television, and the Modern Event. New York / London, 1-14.

TRIMBORN, JÜRGEN (1998): Der deutsche Heimatfilm der fünfziger Jahre. Motive, Symbole und Handlungsmuster. Köln.

WAGNER, MONIKa (1989): Allegorie und Geschichte. Ausstattungsprogramme öffentlicher Gebäude des 19. Jahrhunderts in Deutschland. Von der Cornelius-Schulze zur Malerei der Wilhelminischen Ära. Tübingen.

WAPPENSChMidT, HeInZ-Toni (1984): Allegorie, Symbol und Historienbild im späten 19. Jahrhundert. München, 71-75.

Welzer, Harald (2005): Das kommunikative Gedächtnis. Eine Theorie der Erinnerung. München.

Welzer, Harald / Moller, Sabine / Tschuggnall, Karoline (2002): ,, Opa war kein Nazi “. Nationalsozialismus und Holocaust im Familiengedächtnis. Frankfurt a.M.

WenK, SiLKe (1996): Versteinerte Weiblichkeit. Allegorien in der Skulptur der Moderne. Köln / Weimar / Wien.

ZECHNER, ANKE (2006): Das Affektbild als Stillstand der Narration: Überlegungen zur Schlussszene von Vive L'Amor - Es lebe die Liebe. In: Krause-Wahl, AntJE / Oehlschlägel, Heike / Wiemer, SerJoscha (eds.): Affekte. Analysen ästhetischmedialer Prozesse. Bielefeld, 155-167. 
Filmallegorien der Nationen. Über die Position der Zuschauenden

(C) by the author, licensee Lodz University - Lodz University Press, Lodz, Poland. This article is an open access article distributed under the terms and conditions of the Creative Commons Attribution license CC BY-NC-ND 4.0 (https://creativecommons.org/licenses/by-nc-nd/4.0/) 